Bull. Korean Math. Soc. 52 (2015), No. 2, pp. 453-465

http://dx.doi.org/10.4134/BKMS.2015.52.2.453

\title{
ON POLARS OF MIXED COMPLEX PROJECTION BODIES
}

\author{
Lijuan Liu, Wei Wang, and Qingzhong Huang
}

ABstract. In this paper we establish general Minkowski inequality, Aleksandrov-Fenchel inequality and Brunn-Minkowski inequality for polars of mixed complex projection bodies.

\section{Introduction}

The projection body of a convex body in $\mathbb{R}^{n}$ is one of the central notions that Minkowski introduced within convex geometry. Projection bodies and their polars have received considerable attention over the past few decades (see $[3,5,7,8,10,14,15,16,22,25,26,29,32,33,34,35]$ ). Important volume inequalities for the polars of projection bodies are the Petty projection inequality [23] and the Zhang projection inequality [31]: Among bodies of given volume the polar projection bodies have maximal volume precisely for ellipsoids and minimal volume precisely for simplices. The corresponding results for the volume of the projection body itself are major open problems in convex geometry (see [20]).

Mixed projection bodies in $\mathbb{R}^{n}$ were introduced in the classic volume of Bonnesen-Fenchel [4]. They are related to ordinary projection bodies in the same way that mixed volumes are related to ordinary volumes. In [17] and [18] Lutwak considered the volume of mixed projection bodies and their polars and established analogs of the classical mixed volume inequalities. More inequalities for polars mixed projection bodies were obtained by Leng et al. [14].

The theory of real convex bodies goes back to ancient times and continues to be a very active field now. Until recently the situation with complex convex bodies began to attract attention (see $[1,2,9,11,12,13,24,34,36,37]$ ).

The real vector space $\mathbb{R}^{n}$ of real dimension $n$ is replaced by a complex vector space $\mathbb{C}^{n}$ of dimension $n$. We denote by $\|\cdot\|_{K}$ the norm corresponding to the

Received October 26, 2013; Revised April 4, 2014.

2010 Mathematics Subject Classification. 52A40, 52A20.

Key words and phrases. polar, convex body, mixed complex projection bodies.

Supported in part by Scientific Research Fund of Hunan Provincial Education Department (11C0542) and the National Natural Science Foundations of China (NO. 11326075 and 11071156). 
complex convex body $K \in \mathbb{C}^{n}$ :

$$
K=\left\{z \in \mathbb{C}^{n}:\|z\|_{K} \leq 1\right\} .
$$

In order to define volume, we identify $\mathbb{C}^{n}$ with $\mathbb{R}^{2 n}$ using the standard mapping $(1.2) \quad \xi=\left(\xi_{1}, \ldots, \xi_{n}\right)=\left(\xi_{11}+i \xi_{12}, \ldots, \xi_{n 1}+i \xi_{n 2}\right) \mapsto\left(\xi_{11}, \xi_{12}, \ldots, \xi_{n 1}, \xi_{n 2}\right)$.

The unit ball $B$ in $\mathbb{C}^{n}$ is given by

$$
B=\left\{\xi \in \mathbb{C}^{n}: \sum_{i=1}^{n}\left(\xi_{i 1}^{2}+\xi_{i 2}^{2}\right) \leq 1\right\}
$$

The volume of the unit ball $B$ in $\mathbb{C}^{n}$ is denoted by $\omega_{2 n}$.

Let $K_{1}, \ldots, K_{2 n-1}$ be convex bodies in $\mathbb{C}^{n}$ and $C \subset \mathbb{C}$ be a convex subset. The mixed complex projection body $\Pi^{C}\left(K_{1}, \ldots, K_{2 n-1}\right)$ is the convex body whose support function is defined by (see [2])

(1.3) $h\left(\Pi^{C}\left(K_{1}, \ldots, K_{2 n-1}\right), w\right)=\frac{1}{2 n} \int_{S^{2 n-1}} h(C \cdot w, \xi) d S\left(K_{1}, \ldots, K_{2 n-1}, \xi\right)$, where $C \cdot w:=\{c w \mid c \in C\}$ and $w \in \mathbb{C}^{n}$.

If $C=\{c\}(c \in \mathbb{C})$ is just a point, then $\Pi^{C}\left(K_{1}, \ldots, K_{2 n-1}\right)=\{0\}$. Indeed, for every $w \in \mathbb{C}^{n}$,

$$
\begin{aligned}
h\left(\Pi^{C}\left(K_{1}, \ldots, K_{2 n-1}\right), w\right) & =\frac{1}{2 n} \int_{S^{2 n-1}} h(c w, \xi) d S\left(K_{1}, \ldots, K_{2 n-1}, \xi\right) \\
& =\frac{1}{2 n} \int_{S^{2 n-1}} c w \cdot \xi d S\left(K_{1}, \ldots, K_{2 n-1}, \xi\right) \\
& =\frac{1}{2 n} c w \cdot \int_{S^{2 n-1}} \xi d S\left(K_{1}, \ldots, K_{2 n-1}, \xi\right) \\
& =0,
\end{aligned}
$$

since the centroid of the mixed surface area measure is the origin (see [6]). Thus, $\Pi^{C}\left(K_{1}, \ldots, K_{2 n-1}\right)=\{0\}$. Throughout this article, we assume that $C$ is not a point.

If $K_{1}=\cdots=K_{2 n-i-1}=K$ and $M=\left(K_{2 n-i}, \ldots, K_{2 n-1}\right)$, then the mixed projection body $\Pi^{C}\left(K, \ldots, K, K_{2 n-i}, \ldots, K_{2 n-1}\right)$ is written as $\Pi_{i}^{C}(K, M)$. In particular, we write $\Pi_{i}^{C}(K, L)$ for the mixed complex projection body $\Pi^{C}(K, \ldots, K, L, \ldots, L)$ with $i$ copies of $L$ and $2 n-i-1$ copies of $K$. For the mixed complex projection body $\Pi_{i}^{C}(K, B)$, we simply write $\Pi_{i}^{C} K$. The mixed complex projection body $\Pi_{0}^{C} K$ is written as $\Pi^{C} K$.

Recently, Abardia and Bernig [2] established the general Minkowski inequality, Aleksandrov-Fenchel inequality and Brunn-Minkowski inequality for mixed complex projection bodies. The results can be stated as follows.

Theorem $\mathbf{A}([2])$. If $K$ and $L$ are convex bodies in $\mathbb{C}^{n}$ and $C \subset \mathbb{C}$ is a convex subset, then

$$
V\left(\Pi_{1}^{C}(K, L)\right)^{2 n-1} \geq V\left(\Pi^{C} K\right)^{2 n-2} V\left(\Pi^{C} L\right),
$$

with equality if and only if $K$ and $L$ are homothetic. 
Theorem B ([2]). If $K_{1}, \ldots, K_{2 n-1}$ are convex bodies in $\mathbb{C}^{n}$ and $C \subset \mathbb{C}$ is a convex subset, while $2 \leq k \leq 2 n-2$, then

$$
V\left(\Pi^{C}\left(K_{1}, \ldots, K_{2 n-1}\right)\right)^{k} \geq \prod_{j=1}^{k} V\left(\Pi^{C}(\underbrace{K_{j}, \ldots, K_{j}}_{k}, K_{k+1}, \ldots, K_{2 n-1}) .\right.
$$

Theorem $\mathbf{C}([2])$. If $K$ and $L$ are convex bodies in $\mathbb{C}^{n}$ and $C \subset \mathbb{C}$ is a convex subset, then

$$
V\left(\Pi^{C}(K+L)\right)^{\frac{1}{2 n(2 n-1)}} \geq V\left(\Pi^{C} K\right)^{\frac{1}{2 n(2 n-1)}}+V\left(\Pi^{C} L\right)^{\frac{1}{2 n(2 n-1)}},
$$

with equality if and only if $K$ and $L$ are homothetic.

The main purpose of this article is to establish polar forms of the above three inequalities.

Theorem 1.1. If $K$ and $L$ are convex bodies in $\mathbb{C}^{n}$ and $C \subset \mathbb{C}$ is an origin symmetric convex subset, then

$$
V\left(\Pi_{1}^{C *}(K, L)\right)^{2 n-1} \leq V\left(\Pi^{C *} K\right)^{2 n-2} V\left(\Pi^{C *} L\right),
$$

with equality if and only if $K$ and $L$ are homothetic.

Theorem 1.2. If $K_{1}, \ldots, K_{2 n-1}$ are convex bodies in $\mathbb{C}^{n}$ and $C \subset \mathbb{C}$ is an origin symmetric convex subset, while $2 \leq k \leq 2 n-2$, then

$$
V\left(\Pi^{C *}\left(K_{1}, \ldots, K_{2 n-1}\right)\right)^{k} \leq \prod_{j=1}^{k} V\left(\Pi^{C *}(\underbrace{K_{j}, \ldots, K_{j}}_{k}, K_{k+1}, \ldots, K_{2 n-1}) .\right.
$$

Theorem 1.3. If $K$ and $L$ are convex bodies in $\mathbb{C}^{n}$ and $C \subset \mathbb{C}$ is an origin symmetric convex subset, then

$$
V\left(\Pi^{C *}(K+L)\right)^{-\frac{1}{2 n(2 n-1)}} \geq V\left(\Pi^{C *} K\right)^{-\frac{1}{2 n(2 n-1)}}+V\left(\Pi^{C *} L\right)^{-\frac{1}{2 n(2 n-1)}},
$$

with equality if and only if $K$ and $L$ are homothetic.

Please see the next section for the above interrelated notations, definitions and their background materials.

\section{Notations and background material}

In this section some notations and basic facts about convex bodies are presented. For general references the reader may wish to consult the books of Gardner [7] and Schneider [26].

For $x, y \in \mathbb{R}^{n}$, we denote their scalar product by $x \cdot y$. Similarly, for $x, y \in \mathbb{C}^{n}$, we denote their complex scalar product by $x \cdot y$. Let $\mathcal{K}^{n}$ denote the space of non-empty compact convex bodies in real vector space $\mathbb{R}^{n}$ with the Hausdorff topology. A compact, convex set $K \in \mathcal{K}^{n}$ is uniquely determined by its support function $h(K, \cdot)$ on the unit sphere $S^{n-1}$, defined by

$$
h(K, u)=\max \{x \cdot u: x \in K\}, u \in S^{n-1},
$$


where $x \cdot u$ denotes the scalar product $x$ and $u$.

A compact set $K \in \mathbb{R}^{n}$ is called a star body if the origin is an interior point of $K$, every straight line passing through the origin crosses the boundary of the set at exactly two points, and its radial function $\rho(K, \cdot)$ defined by

$$
\rho(K, u)=\max \{\lambda: \lambda u \in K\}, u \in S^{n-1}
$$

is positive and continuous on $S^{n-1}$. Let $\mathcal{S}^{n}$ denote the space of the star bodies in real vector space $\mathbb{R}^{n}$.

We recall that the polar coordinate formula for volume in $\mathbb{R}^{n}$ is

$$
V(K)=\frac{1}{n} \int_{S^{n-1}} \rho(K, u)^{n} d S(u)
$$

where $d S(u)$ denotes the spherical Lebesgue measure on $S^{n-1}$.

If $K$ is a convex body that contains the origin in its interior, the polar body of $K, K^{*}$, is defined by

$$
K^{*}:=\left\{x \in \mathbb{R}^{n}: x \cdot y \leq 1 \text { for all } y \in K\right\} .
$$

From the definition of support function, for every convex body $K$ that contains the origin in its interior and $\lambda>0$, it follows that

$$
\left(K^{*}\right)^{*}=K \text { and }(\lambda K)^{*}=\frac{1}{\lambda} K^{*} .
$$

In particular, if $K$ is a convex body that contains the origin in its interior, then

$$
h\left(K^{*}, u\right)=\frac{1}{\rho(K, u)} \text { and } \rho\left(K^{*}, u\right)=\frac{1}{h(K, u)}, u \in S^{n-1} .
$$

For $K_{1}, K_{2} \in \mathcal{K}^{n}$ and $\lambda_{1}, \lambda_{2} \geq 0$, the Minkowski addition $\lambda_{1} K_{1}+\lambda_{2} K_{2}$ is the convex body defined by

$$
h\left(\lambda_{1} K_{1}+\lambda_{2} K_{2}, \cdot\right)=\lambda_{1} h\left(K_{1}, \cdot\right)+\lambda_{2} h\left(K_{2}, \cdot\right) .
$$

If $K_{i} \in \mathcal{K}^{n}(i=1,2, \ldots, k)$ and $\lambda_{i}(i=1,2, \ldots, k)$ are nonnegative real numbers, then the volume of $\lambda_{1} K_{1}+\cdots+\lambda_{k} K_{k}$ is a homogeneous polynomial of degree $n$ in $\lambda_{i}$ given by

$$
V\left(\lambda_{1} K_{1}+\cdots+\lambda_{k} K_{k}\right)=\sum_{i_{1}, \ldots, i_{n}} V\left(K_{i_{1}}, \ldots, K_{i_{n}}\right) \lambda_{i_{1}} \cdots \lambda_{i_{n}},
$$

where the sum is taken over all $n$-tuples $\left(i_{1}, \ldots, i_{n}\right)$ of positive integers not exceeding $m$. The coefficient $V\left(K_{i_{1}}, \ldots, K_{i_{n}}\right)$ is called the mixed volume of $K_{i_{1}}, \ldots, K_{i_{n}}$. And it is nonnegative, symmetric in its arguments and monotone (with respect to set inclusion in each component). In particular, $V(K, \ldots, K)=$ $V(K)$. Let $K_{1}=\cdots=K_{n-i}=K$ and $K_{n-i+1}=\cdots=K_{n}=L$, the mixed volume $V\left(K_{1}, \ldots, K_{n}\right)$ is usually written as $V_{i}(K, L)$. If $L=B, V_{i}(K, B)$ is the $i$-th Quermassintegral of $K$ and is written as $W_{i}(K)$. For $0 \leq i \leq n-1$, we write $W_{i}(K, L)$ for the mixed volume $V(\underbrace{K, \ldots, K}_{n-i-1}, \underbrace{B, \ldots, B}_{i}, L)$. 
The mixed volume $V\left(K_{1}, \ldots, K_{n}\right)$ has the following integral representation:

$$
V\left(K_{1}, \ldots, K_{n}\right)=\frac{1}{n} \int_{S^{n-1}} h\left(K_{n}, u\right) d S\left(K_{1}, \ldots, K_{n-1}, u\right)
$$

where $S\left(K_{1}, \ldots, K_{n-1}, \cdot\right)$ denotes the mixed surface area measure.

One of the most general and fundamental inequalities for mixed volumes is the Aleksandrov-Fenchel inequality: If $K_{1}, \ldots, K_{n} \in \mathcal{K}^{n}$ and $1 \leq k \leq n$, then

$$
V\left(K_{1}, \ldots, K_{n}\right)^{k} \geq \prod_{j=1}^{k} V(\underbrace{K_{j}, \ldots, K_{j}}_{k}, K_{k+1}, \ldots, K_{n}) .
$$

Unfortunately, the equality conditions of this inequality are, in general, unknown.

An important special case of inequality (2.7), where the equality conditions are known, is the classical inequality between the quermassintegrals (see [18]): If $K \in \mathcal{K}^{n}$, and $0 \leq i<j<n$, then

$$
\omega_{n}^{j-i} W_{i}(K)^{n-j} \leq W_{j}(K)^{n-i}
$$

with equality if and only if $K$ is a ball.

The Minkowski inequality for mixed volumes states as follows (see [19]): If $K, L \in \mathcal{K}^{n}$ and $0 \leq i \leq n-2$, then

$$
W_{i}(K, L)^{n-i} \geq W_{i}(K)^{n-i-1} W_{i}(L),
$$

with equality if and only if $K$ and $L$ are homothetic.

A consequence of the Minkowski inequality is the following Brunn-Minkowski inequality: If $K, L \in \mathcal{K}^{n}$ and $0 \leq i \leq n-2$, then

$$
W_{i}(K+L)^{\frac{1}{n-i}} \geq W_{i}(K)^{\frac{1}{n-i}}+W_{i}(L)^{\frac{1}{n-i}},
$$

with equality if and only if $K$ and $L$ are homothetic.

A generalization of inequality $(2.10)$ is also known (but without equality conditions): If $K, L, K_{1}, \ldots, K_{i} \in \mathcal{K}^{n}, 0 \leq i \leq n-2$, and $M=\left(K_{1}, \ldots, K_{i}\right)$, then

$$
V_{i}(K+L, M)^{\frac{1}{n-i}} \geq V_{i}(K, M)^{\frac{1}{n-i}}+V_{i}(L, M)^{\frac{1}{n-i}} .
$$

The dual mixed volume $\widetilde{V}_{-1}(K, L)$ of $K, L \in \mathcal{S}^{n}$ was defined by (see [21])

$$
\widetilde{V}_{-1}(K, L)=\frac{1}{n} \int_{S^{n-1}} \rho(K, u)^{n+1} \rho(L, u)^{-1} d S(u) .
$$

It is easy to check that

$$
\widetilde{V}_{-1}(K, K)=V(K)
$$

The following Minkowski inequality for dual mixed volume $\widetilde{V}_{-1}(K, L)$ will play an important role in our proof (see [21]). If $K, L \in \mathcal{S}^{n}$, then

$$
\widetilde{V}_{-1}(K, L)^{n} \geq V(K)^{n+1} V(L)^{-1}
$$

with equality if and only if $K$ and $L$ are dilates. 
Let $K_{1}, \ldots, K_{2 n-1}$ be convex bodies in $\mathbb{C}^{n}$. If $C$ is an origin symmetric convex subset in $\mathbb{C}$, then the mixed complex projection body $\Pi^{C}\left(K_{1}, \ldots, K_{2 n-1}\right)$ is origin symmetric. Note that origin symmetric complex convex bodies in $\mathbb{C}^{n}$ correspond to those origin symmetric convex bodies $K$ in $\mathbb{R}^{2 n}$ that are invariant with respect to any coordinate-wise two-dimensional rotation, namely for each $\theta \in[0,2 \pi]$ and each $\xi=\left(\xi_{11}, \xi_{12}, \ldots, \xi_{n 1}, \xi_{n 2}\right) \in \mathbb{R}^{2 n}$,

$$
\|\xi\|_{K}=\left\|R_{\theta}\left(\xi_{11}, \xi_{12}\right), \ldots, R_{\theta}\left(\xi_{n 1}, \xi_{n 2}\right)\right\|_{K}
$$

where $R_{\theta}$ stands for the counterclockwise rotation of $\mathbb{R}^{2}$ by the angle $\theta$ with respect to the origin.

We use $\Pi^{C *}\left(K_{1}, \ldots, K_{2 n-1}\right)$ to denote the polar body of $\Pi^{C}\left(K_{1}, \ldots, K_{2 n-1}\right)$, and call it a polar of mixed complex projection body $K_{i}(i=1, \ldots, 2 n-1)$. We will simply write $\Pi_{i}^{C *} K$ and $\Pi_{i}^{C *}(K, L)$ rather than $\left(\Pi_{i}^{C} K\right)^{*}$ and $\left(\Pi_{i}^{C}(K, L)\right)^{*}$, respectively.

Let $\mathcal{C}\left(S^{n-1}\right)$ be the spaces of continuous functions on $S^{n-1}$ with uniform topology and let $\mathcal{M}\left(S^{n-1}\right)$ denote the dual space of signed finite Borel measures with weak* topology. The convolution $\mu * f \in \mathcal{C}\left(S^{n-1}\right)$ of a measure $\mu \in$ $\mathcal{M}\left(S^{n-1}\right)$ and a function $f \in \mathcal{C}\left(S^{n-1}\right)$ is defined by:

$$
(\mu * f)(u)=\int_{S^{n-1}} f(u \cdot) d \mu(\cdot) .
$$

The canonical pairing of $f \in \mathcal{C}\left(S^{n-1}\right)$ and $\mu \in \mathcal{M}\left(S^{n-1}\right)$ is defined by:

$$
\langle\mu, f\rangle=\langle f, \mu\rangle=\int_{S^{n-1}} f(u) d \mu(u) .
$$

The following property of spherical convolution will be very useful (see [28]): If $\mu, \nu \in \mathcal{M}\left(S^{n-1}\right)$ and $f \in \mathcal{C}\left(S^{n-1}\right)$, then

$$
\langle\mu * \nu, f\rangle=\langle\mu, f * \nu\rangle \text {. }
$$

\section{Main results}

Lemma 3.1 ([2]). If $K_{1}, \ldots, K_{2 n-1}, L_{1}, \ldots, L_{2 n-1}$ are convex bodies in $\mathbb{C}^{n}$ and $C \subset \mathbb{C}$ is a convex subset, then

$$
\begin{aligned}
& V\left(K_{1}, \ldots, K_{2 n-1}, \Pi^{C}\left(L_{1}, \ldots, L_{2 n-1}\right)\right) \\
= & V\left(L_{1}, \ldots, L_{2 n-1}, \Pi^{\bar{C}}\left(K_{1}, \ldots, K_{2 n-1}\right)\right),
\end{aligned}
$$

where $\bar{C}$ is the complex conjugate of $C$.

Note that $h(\bar{C} \cdot w, \xi)=h(C \cdot \xi, w)$ and the surface area measure $S(B, \cdot)$ is constant in $S^{2 n-1}$, we have the following lemma.

Lemma 3.2 ([30]). If $C \subset \mathbb{C}$ is a convex subset, then

$$
\Pi^{C}(B, \ldots, B)=\Pi^{\bar{C}}(B, \ldots, B)=r_{C} B,
$$

where $r_{C}$ is a constant which depends only on $C$. 
Combine the special case $K_{1}=\cdots=K_{2 n-1}=B$ of Lemmas 3.1 and 3.2 to get:

Lemma 3.3. If $L_{1}, \ldots, L_{2 n-1}$ are convex bodies in $\mathbb{C}^{n}$ and $C \subset \mathbb{C}$ is a convex subset, then

$$
W_{2 n-1}\left(\Pi^{C}\left(L_{1}, \ldots, L_{2 n-1}\right)\right)=r_{C} V\left(L_{1}, \ldots, L_{2 n-1}, B\right) .
$$

For $L_{1}=\cdots=L_{2 n-2}=K$ and $L_{2 n-1}=L$, identity (3.1) becomes

$$
W_{2 n-1}\left(\Pi_{1}^{C}(K, L)\right)=r_{C} W_{1}(K, L) .
$$

For $L_{1}=\cdots=L_{2 n-i-1}=L$ and $L_{2 n-i}=\cdots=L_{2 n-1}=B$, identity (3.1) becomes,

$$
W_{2 n-1}\left(\Pi_{i}^{C} L\right)=r_{C} W_{i+1}(L) .
$$
by

In [24], Schuster introduced the operator $M_{\Phi}: \mathcal{S}^{n} \rightarrow \mathcal{K}^{n}$ which was defined

$$
h\left(M_{\Phi} L, u\right)=\rho^{n+1}(L, \cdot) * h(F, \cdot), u \in S^{n-1} .
$$

Here $h(F, \cdot)$ is the generating function of $\Phi$, where $F \in \mathbb{R}^{n}$ is a figure of revolution which is not a singleton and depends on $u$.

In particular, for the classical projection body operator $\Pi: \mathcal{K}^{n} \rightarrow \mathcal{K}^{n}$, the operator $M_{\Pi}: \mathcal{S}^{n} \rightarrow \mathcal{K}^{n}$ is defined by

$$
h\left(M_{\Pi} K, u\right)=\rho^{n+1}(K, \cdot) * h\left(\left[-\frac{1}{2}, \frac{1}{2}\right] \cdot u, \cdot\right), u \in S^{n-1},
$$

where $F=\left[-\frac{1}{2}, \frac{1}{2}\right] \cdot u=\left[-\frac{u}{2}, \frac{u}{2}\right]$.

This operator can be extended to complex case. Let $L$ be a star body in $\mathbb{C}^{n}$ and $C$ be an origin symmetric convex set in $\mathbb{C}$. Then the operator $M_{\Pi^{C}}$ can be defined by

$$
h\left(M_{\Pi^{C}} L, \xi\right)=\rho^{2 n+1}(L, \cdot) * h(C \cdot \xi, \cdot), \xi \in \mathbb{C}^{n},
$$

where $F=C \cdot \xi$.

Lemma 3.4. Let $K_{1}, \ldots, K_{2 n-1}$ be convex bodies and $L$ be a star body in $\mathbb{C}^{n}$. If $C$ is an origin symmetric convex set in $\mathbb{C}$, then

$$
\widetilde{V}_{-1}\left(L, \Pi^{C *}\left(K_{1}, \ldots, K_{2 n-1}\right)\right)=V\left(K_{1}, \ldots, K_{2 n-1}, M_{\Pi \bar{C}} L\right) .
$$

Proof. By (2.12), (2.5), (1.3), (3.4), (2.17) and (2.6), we have

$$
\begin{aligned}
& \widetilde{V}_{-1}\left(L, \Pi^{C *}\left(K_{1}, \ldots, K_{2 n-1}\right)\right) \\
= & \frac{1}{2 n} \int_{S^{2 n-1}} \rho(L, \xi)^{2 n+1} \rho\left(\Pi^{C *}\left(K_{1}, \ldots, K_{2 n-1}\right), \xi\right)^{-1} d \xi \\
= & \frac{1}{2 n} \int_{S^{2 n-1}} \rho(L, \xi)^{2 n+1} h\left(\Pi^{C}\left(K_{1}, \ldots, K_{2 n-1}\right), \xi\right) d \xi \\
= & \frac{1}{(2 n)^{2}}\left\langle\rho(L, \xi)^{2 n+1}, h(C \cdot \xi, \cdot) * S\left(K_{1}, \ldots, K_{2 n-1}, \cdot\right)\right\rangle
\end{aligned}
$$




$$
\begin{aligned}
& =\frac{1}{(2 n)^{2}}\left\langle\rho(L, \cdot)^{2 n+1} * h(\bar{C} \cdot \omega, \cdot), S\left(K_{1}, \ldots, K_{2 n-1}, \omega\right)\right\rangle \\
& =\frac{1}{2 n}\left\langle h\left(M_{\Pi_{\bar{C}}} L, \omega\right), S\left(K_{1}, \ldots, K_{2 n-1}, \omega\right)\right\rangle \\
& =\frac{1}{2 n} \int_{S^{2 n-1}} h\left(M_{\Pi_{\bar{C}}} L, \omega\right) d S\left(K_{1}, \ldots, K_{2 n-1}, \omega\right) \\
& =V\left(K_{1}, \ldots, K_{2 n-1}, M_{\Pi_{\bar{C}}} L\right) .
\end{aligned}
$$

If $K_{1}=\cdots=K_{2 n-i-1}=K$ and $K_{2 n-i}=\cdots=K_{2 n-1}=B$, then Lemma 3.4 reduces to:

Lemma 3.5. Let $K$ be a convex body and $L$ be a star body in $\mathbb{C}^{n}$. If $C$ is an origin symmetric convex set in $\mathbb{C}$, then

$$
\widetilde{V}_{-1}\left(L, \Pi_{i}^{C *} K\right)=W_{i}\left(K, M_{\Pi^{C}} L\right) .
$$

Proof of Theorem 1.1. Suppose $Q$ is a star body in $\mathbb{C}^{n}$, from Lemma 3.4 and the Aleksandrov-Fenchel inequality (2.7) and the Minkowski inequality (2.14), it follows that

$$
\begin{aligned}
& \widetilde{V}_{-1}\left(Q, \Pi_{1}^{C *}(K, L)\right)^{2 n-1} \\
= & V\left(K, \ldots, K, L, M_{\Pi^{\bar{C}}} Q\right)^{2 n-1} \\
\geq & V_{1}\left(K, M_{\Pi^{C}} Q\right)^{2 n-2} V_{1}\left(L, M_{\Pi^{\bar{C}}} Q\right) \\
= & \widetilde{V}_{-1}\left(Q, \Pi^{C *} K\right)^{2 n-2} \widetilde{V}_{-1}\left(Q, \Pi^{C *} L\right) \\
\geq & V(Q)^{\frac{(2 n-1)(2 n+1)}{2 n}} V\left(\Pi^{C *} K\right)^{-\frac{2 n-2}{2 n}} V\left(\Pi^{C *} L\right)^{-\frac{1}{2 n}} .
\end{aligned}
$$

By the equality conditions of (2.14), equality in (3.5) holds if and only if $Q$, $\Pi^{C *} K$, and $\Pi^{C *} L$ are dilates.

Set $Q=\Pi_{1}^{C *}(K, L)$ and note that $\widetilde{V}_{-1}(Q, Q)=V(Q)$ to obtain the desired inequality (1.4). If there is equality in (1.4), then there exist $\lambda_{1}, \lambda_{2}>0$ such that

$$
\Pi_{1}^{C *}(K, L)=\lambda_{1} \Pi^{C *} K=\lambda_{2} \Pi^{C *} L .
$$

From equality in (1.4), it follows that

$$
\lambda_{1}^{2 n-2} \lambda_{2}=1 .
$$

On the other hand, from the definition of the polar body, (3.6) is equivalent to

$$
\Pi_{1}^{C}(K, L)=\frac{1}{\lambda_{1}} \Pi^{C} K=\frac{1}{\lambda_{2}} \Pi^{C} L .
$$

Moreover, (3.2), (3.3) and (3.8) imply

$$
\lambda_{1}=\frac{W_{1}(K)}{W_{1}(K, L)} \text { and } \lambda_{2}=\frac{W_{1}(L)}{W_{1}(K, L)} .
$$


Hence, by (3.7) and (3.9) we have

$$
W_{1}(K, L)^{2 n-1}=W_{1}(K)^{2 n-2} W_{1}(L),
$$

which implies, by (2.9), that $K$ and $L$ are homothetic.

Remark 1. The real case of Theorem 1.1 was given by Zhao and Leng [35]. The real case of Theorem 1.1 for the polar Blaschke-Minkowski homomorphisms was given by Schuster [27].

Proof of Theorem 1.2. Suppose $Q$ is a star body in $\mathbb{C}^{n}$, from Lemma 3.4 and the Aleksandrov-Fenchel inequality (2.7), we have that

$$
\begin{aligned}
& \widetilde{V}_{-1}\left(Q, \Pi^{C *}\left(K_{1}, \ldots, K_{2 n-1}\right)\right)^{k} \\
= & V\left(K_{1}, \ldots, K_{2 n-1}, M_{\Pi \bar{C}} Q\right)^{k} \\
\geq & \prod_{j=1}^{k} V(\underbrace{K_{j}, \ldots, K_{j}}_{k}, K_{k+1}, \ldots, K_{2 n-1}, M_{\Pi \bar{C}} Q) \\
= & \prod_{j=1}^{k} \widetilde{V}_{-1}\left(Q, \Pi^{C *}(\underbrace{K_{j}, \ldots, K_{j}}_{k}, K_{k+1}, \ldots, K_{2 n-1})\right) .
\end{aligned}
$$

Write $\Pi_{k^{\prime}}^{C *}\left(K_{j}, N\right)$ for the mixed operator $\Pi^{C *}\left(K_{j}, \ldots, K_{j}, K_{k+1}, \ldots, K_{2 n-1}\right)$, where $k^{\prime}=2 n-k-1$. Then by inequality (2.14), we have

$$
\widetilde{V}_{-1}\left(Q, \Pi_{k^{\prime}}^{C *}\left(K_{j}, N\right)\right)^{2 n} \geq V(Q)^{2 n+1} V\left(\Pi_{k^{\prime}}^{C *}\left(K_{j}, N\right)\right)^{-1} .
$$

Hence, we obtain

$$
\widetilde{V}_{-1}\left(Q, \Pi^{C *}\left(K_{1}, \ldots, K_{2 n-1}\right)\right)^{2 n k} \geq V(Q)^{(2 n+1) k} \prod_{j=1}^{k} V\left(\Pi_{k^{\prime}}^{C *}\left(K_{j}, N\right)\right)^{-1} .
$$

Setting $Q=\Pi^{C *}\left(K_{1}, \ldots, K_{2 n-1}\right)$ in (3.10), it becomes the desired inequality.

Remark 2. The real case of Theorem 1.2 was given by Zhao and Leng [35]. The real case of Theorem 1.2 for the polar Blaschke-Minkowski homomorphisms was given by Schuster [27].

Combine the special case $k=2 n-2$ of Theorem 1.2 and Theorem 1.1 to obtain:

Corollary 3.6. If $K_{1}, \ldots, K_{2 n-1}$ are convex bodies in $\mathbb{C}^{n}$ and $C \subset \mathbb{C}$ is an origin symmetric convex subset in $\mathbb{C}$, then

$$
V\left(\Pi^{C *}\left(K_{1}, \ldots, K_{2 n-1}\right)\right)^{2 n-1} \leq V\left(\Pi^{C *} K_{1}\right) \cdots V\left(\Pi^{C *} K_{2 n-1}\right),
$$

with equality if and only if the $K_{j}$ are homothetic. 
The special case $K_{1}=\cdots=K_{2 n-j-1}=K$ and $K_{2 n-j}=\cdots=K_{2 n-1}=L$ of Corollary 3.6 leads to a generalization of Theorem 1.1:

Corollary 3.7. If $K$ and $L$ are convex bodies in $\mathbb{C}^{n}$ and $C \subset \mathbb{C}$ is an origin symmetric convex subset in $\mathbb{C}$, while $1 \leq j \leq 2 n-2$, then

$$
V\left(\Pi_{j}^{C *}(K, L)\right)^{2 n-1} \leq V\left(\Pi^{C *} K\right)^{2 n-j-1} V\left(\Pi^{C *} L\right)^{j},
$$

with equality if and only if $K$ and $L$ are homothetic.

An important consequence of Corollary 3.7 states as follows.

Theorem 3.8. Let $K, L$ be convex bodies in $\mathbb{C}^{n}$ and $\mathcal{M} \subset \mathbb{C}^{n}$ be a subset which contains $K$ and $L$. Suppose $C \subset \mathbb{C}$ is a convex subset and $1 \leq j \leq 2 n-2$. If either

$$
V\left(\Pi_{j}^{C *}(K, Q)\right)=V\left(\Pi_{j}^{C *}(L, Q)\right) \text { for all } Q \in \mathcal{M}
$$

or

$$
V\left(\Pi_{j}^{C *}(Q, K)\right)=V\left(\Pi_{j}^{C *}(Q, L)\right) \text { for all } Q \in \mathcal{M},
$$

hold, then it follows that $K=L$, up to translation.

Proof. Suppose (3.11) holds. Take $K$ for $Q$ in (3.11), use Corollary 3.7 to get

$$
V\left(\Pi^{C *} K\right) \leq V\left(\Pi^{C *} L\right),
$$

with equality if and only if $K$ and $L$ are homothetic.

Take $L$ for $Q$ in (3.11), use Corollary 3.7 to get

$$
V\left(\Pi^{C *} K\right) \geq V\left(\Pi^{C *} L\right) .
$$

Hence, there is equality in (3.13) and thus, there is a $\lambda>0$ for which $K$ and $\lambda L$ are translates. Note that the complex projection body operator $\Pi^{C}$ is homogeneous of degree $2 n-1$. But equality in (3.13) implies that $\lambda=1$.

Exactly the same sort of argument shows that condition (3.12) implies that $K$ and $L$ must be translates.

Remark 3. The real case of Theorem 3.8 was given by Zhao and Leng [35].

In fact a considerably more general inequality of Brunn-Minkowski inequality for polars of mixed complex projection bodies holds:

Theorem 3.9. If $K$ and $L$ are convex bodies in $\mathbb{C}^{n}$ and $C \subset \mathbb{C}$ is an origin symmetric complex convex subset, while $0 \leq j \leq 2 n-2$, then

$$
V\left(\Pi_{j}^{C *}(K+L)\right)^{-\frac{1}{2 n(2 n-j-1)}} \leq V\left(\Pi_{j}^{C *} K\right)^{-\frac{1}{2 n(2 n-j-1)}}+V\left(\Pi_{j}^{C *} L\right)^{-\frac{1}{2 n(2 n-j-1)}},
$$

with equality if and only if $K$ and $L$ are homothetic. 
Proof. Suppose $Q$ is a star body in $\mathbb{C}^{n}$, from Lemma 3.5, (2.11) and (2.14), we have

$$
\begin{aligned}
& \widetilde{V}_{-1}\left(Q, \Pi_{j}^{C *}(K+L)\right)^{\frac{1}{2 n-j-1}} \\
= & W_{j}\left(K+L, M_{\Pi_{\bar{C}}} Q\right)^{\frac{1}{2 n-j-1}} \\
\geq & W_{j}\left(K, M_{\Pi_{\bar{C}}} Q\right)^{\frac{1}{2 n-j-1}}+W_{j}\left(L, M_{\Pi_{\bar{C}}} Q\right)^{\frac{1}{2 n-j-1}} \\
= & \widetilde{V}_{-1}\left(Q, \Pi_{j}^{C *} K\right)^{\frac{1}{2 n-j-1}}+\widetilde{V}_{-1}\left(Q, \Pi_{j}^{C *} L\right)^{\frac{1}{2 n-j-1}} \\
\geq & V(Q)^{\frac{2 n+1}{2 n(2 n-j-1)}}\left[V\left(\Pi_{j}^{C *} K\right)^{-\frac{1}{2 n(2 n-j-1)}}+V\left(\Pi_{j}^{C *} L\right)^{-\frac{1}{2 n(2 n-j-1)}}\right] .
\end{aligned}
$$

By the equality conditions of (2.14), equality in (3.15) holds if and only if $Q, \Pi_{j}^{C *} K$ and $\Pi_{j}^{C *} L$ are delates.

Set $Q=\Pi_{j}^{C *}(K+L)$ and note that $\widetilde{V}_{-1}(Q, Q)=V(Q)$ to obtain the desired inequality (3.14). If there is equality in (3.14), then there exist $\lambda_{1}, \lambda_{2}>0$ such that

$$
\Pi_{j}^{C *}(K+L)=\lambda_{1} \Pi_{j}^{C *} K=\lambda_{2} \Pi_{j}^{C *} L .
$$

From equality in (3.14), it follows that

$$
\lambda_{1}^{\frac{1}{2 n-j-1}}+\lambda_{2}^{\frac{1}{2 n-j-1}}=1 \text {. }
$$

On the other hand, from the definition of polar body, (3.16) is equivalent to

$$
\Pi_{j}^{C}(K+L)=\frac{1}{\lambda_{1}} \Pi_{j}^{C} K=\frac{1}{\lambda_{2}} \Pi_{j}^{C} L .
$$

Moreover, (3.3) and (3.18) imply

$$
\lambda_{1}=\frac{W_{j+1}(K)}{W_{j+1}(K, L)} \text { and } \lambda_{2}=\frac{W_{j+1}(L)}{W_{j+1}(K, L)} .
$$

Hence, by (3.17) and (3.19) we have

$$
W_{j+1}(K+L)^{\frac{1}{2 n-j-1}}=W_{j+1}(K)^{\frac{1}{2 n-j-1}}+W_{j+1}(L)^{\frac{1}{2 n-j-1}}
$$

which implies, by (2.10), that $K$ and $L$ are homothetic.

Remark 4. The case $j=0$ of Theorem 3.9 is just Theorem 1.3. The real case of Theorem 1.3 was given by Schuster [27].

\section{References}

[1] J. Abardia, Difference bodies in complex vector spaces, J. Funct. Anal. 263 (2012), no. $11,3588-3603$.

[2] J. Abardia and A. Bernig, Projection bodies in complex vector spaces, Adv. Math. 211 (2011), no. 2, 830-846.

[3] E. D. Bolker, A class of convex bodies, Trans. Amer. Math. Soc. 145 (1969), 323-345.

[4] T. Bonnesen and W. Fenchel, Theorie der konvexen Kø̈rper, Springer-Verlag, Berlin, 1934.

[5] J. Bourgain and J. Lindenstrauss, Projection bodies, Geometric aspects of functional analysis (1986/87), 250-270, Lecture Notes in Math., 1317, Springer, Berlin, 1988. 
[6] W. Fenchel and B. Jessen, Mengenfunktionen und konvexe Körper, Det. Kgl. Danske Videnskab. Selskab, Mat.-Fys. Medd. 16 (1938), no. 3, 1-31.

[7] R. J. Gardner, Geometric Tomography, Second ed., Cambridge Univ. Press, New York, 2006.

[8] C. Haberl, Minkowski valuations intertwining with the special linear group, J. Eur. Math. Soc. 14 (2012), no. 5, 1565-1597.

[9] Q. Z. Huang, B. W. He, and G. T. Wang, The Busemann theorem for complex p-convex bodies, Arch. Math. 99 (2012), no. 3, 289-299.

[10] A. Koldobsky, Fourier Analysis in Convex Geometry, Math. Surveys Monogr., vol. 116, Amer. Math. Soc., 2005

[11] A. Koldobsky, H. König, and M. Zymonopoulou, The complex Busemann-Petty problem on sections of convex bodies, Adv. Math. 218 (2008), no. 2, 352-367.

[12] A. Koldobsky, G. Paouris, and M. Zymonopoulou, Complex intersection bodies, J. Lond. Math. Soc. 88 (2013), no. 2, 538-562.

[13] A. Koldobsky and M. Zymonopoulou, Extremal sections of complex $l_{p}$-ball, $0<p \leq 2$, Studia Math. 159 (2003), no. 2, 185-194.

[14] G. S. Leng, C. J. Zhao, B. W. He, and X. Y. Li, Inequalities for polars of mixed projection bodies, Sci. China Ser. A 47 (2004), no. 2, 175-186.

[15] M. Ludwig, Projection bodies and valuations, Adv. Math. 172 (2002), no. 2, 158-168.

[16] _ Minkowski areas and valuations, J. Differential Geom. 86 (2010), no. 1, 133161.

[17] E. Lutwak, Mixed projection inequalities, Trans. Amer. Math. Soc. 287 (1985), 91-105.

[18] _ Inequalities for mixed projection bodies, Trans. Amer. Math. Soc. 339 (1993), 901-916.

[19] , The Brunn-Minkowski-Firey theory I: Mixed volumes and the Minkowski problem, J. Differential Geom. 38 (1993), 131-150.

[20] _ Selected affine isoperimetric inequalities, Handbook of convex geometry, Vol. A, B, 151-176, North-Holland, Amsterdam, 1993.

[21] _ The Brunn-Minkowski-Firey theory II: Affine and geominimal surface areas, Adv. Math. 118 (1996), no. 2, 244-294.

[22] C. M. Petty, Projection bodies, Proc. Colloquium on Convexity (Copenhagen, 1965), pp. 234-241, Kobenhavns Univ. Mat. Inst., Copenhagen, 1967.

[23] _ Isoperimetric problems, Proceedings of the Conference on Convexity and Combinatorial Geometry (Univ. Oklahoma, Norman, Okla., 1971), pp. 26-41. Dept. Math., Univ. Oklahoma, Norman, Okla., 1971.

[24] B. Rubin, Comparison of volumes of convex bodies in real, complex, and quaternionic spaces, Adv. Math. 225 (2010), no. 3, 1461-1498.

[25] R. Schneider, Zu einem Problem von Shephard über die Projektionen konvexer Körpor, Math. Z. 101 (1967), 71-82.

[26] _ Convex Bodies: The Brunn-Minkowski Theory, Cambridge Univ. Press, Cambridge, 1993

[27] F. E. Schuster, Volume inequalities and additive maps of convex bodies, Mathematika 53 (2006), no. 2, 211-234

[28] Convolutions and multiplier transformations of convex bodies, Trans. Amer. Math. Soc. 359 (2007), no. 11, 5567-5591.

[29] F. E. Schuster and T. Wannerer, GL $(n)$ contravariant Minkowski valuations, Trans. Amer. Math. Soc. 364 (2012), no. 2, 815-826.

[30] W. Wang and R. G. He, Inequalities for mixed complex projection bodies, Taiwanese J. Math. 17 (2013), no. 6, 1887-1899.

[31] G. Zhang, Centered bodies and dual mixed volumes, Trans. Amer. Math. Soc. 345 (1994), no. $2,777-801$ 
[32] C. J. Zhao, $L_{p}$-dual Quermassintegral sums, Sci. China Ser. A 50 (2007), no. 9, 13471360.

[33] _ On radial and polar Blaschke-Minkowski homomorphisms, Proc. Amer. Math. Soc. 141 (2013), no. 2, 667-676.

[34] Volume differences of mixed complex projection bodies, Bull. Belgian Math. Soc. 21 (2014), no. 3, 553-564.

[35] C. J. Zhao and G. S. Leng, On polars of mixed projection bodies, J. Math. Anal. Appl. 316 (2006), no. 2, 664-678.

[36] M. Zymonopoulou, The complex Busemann-Petty problem for arbitrary measures, Arch. Math. (Basel) 91 (2008), no. 5, 436-449.

[37] The modified complex Busemann-Petty problem on sections of convex bodies, Positivity 13 (2009), no. 4, 717-733.

LiJUAN LIU

Department of Mathematics

ShANGHAi UNIVERSITY

Shanghai 200444, P. R. China

E-mail address: ljliu@shu.edu.cn

WEI WANG

School of Mathematics and Computational Science

Hunan University of Science and Technology

Xiangtan 411201, P. R. China

E-mail address: wwang@hnust.edu.cn

QINGZHONG HuANG

College of mathematics

Physics AND INFORMATION ENGINEERING

JIAXING UNIVERSITY

JiAXING 314001, P. R. ChinA

E-mail address: hqz376560571@163.com 